\title{
DECOMPOSITIONS OF THE CONGRUENCE LATTICE OF A SEMIGROUP
}

\author{
by W. D. MUNN \\ (Received 27th October 1978)
}

The purpose of this note is to extend the results of Reilly and Scheiblich (6) (see also Scheiblich (7) and Hall (2)) on the $\theta$-class decomposition of the congruence lattice of a regular semigroup and, at the same time, to provide an alternative proof of these results.

Except where otherwise stated, the notation and terminology is that of Howie (4).

We begin with some lattice-theoretic definitions. Let $(L, \leqq, \wedge, \vee)$ be a complete lattice. A nonempty subset $S$ of $L$ is called a complete meet [join] subsemilattice of $L$ if and only if, for every nonempty family $\left(a_{i}\right)_{i \in I}$ of elements of $S$,

$$
\bigvee_{i \in I} a_{i} \in S \quad\left[\bigvee_{i \in I} a_{i} \in S\right]
$$

Clearly, every complete meet [join] subsemilattice of $L$ must contain a least [greatest] element under $\leqq$. A nonempty subset of $L$ is called a complete sublattice of $L$ if and only if it is both a complete meet subsemilattice and a complete join subsemilattice of $L$.

An equivalence $\phi$ on $L$ is called a complete meet [join] congruence on $L$ if and only if, for every nonempty family $\left(a_{i}, b_{i}\right)_{i \in I}$ of elements of $\phi$,

$$
\left(\bigvee_{i \in I} a_{i}, \bigvee_{i \in I} b_{i}\right) \in \phi \quad\left[\left(\bigvee_{i \in I} a_{i}, \bigvee_{i \in I} b_{i}\right) \in \phi\right]
$$

Further, $\phi$ is called a complete lattice congruence on $L$ if and only if it is both a complete meet congruence and a complete join congruence. It is readily seen that if $\phi$ is a complete meet [join] congruence on $L$ then each $\phi$-class is a complete meet [join] subsemilattice of $L$.

The set of all congruences on a semigroup $S$ is a complete lattice with respect to inclusion: it will be denoted by $\mathscr{C}(S)$. The meet of any given nonempty family $\left(\rho_{i}\right)_{i \in I}$ of congruences on $S$ is the intersection $\bigcap_{i \in I} \rho_{i}$ while the join $\bigvee_{i \in I} \rho_{i}$ of the family is the least equivalence containing every $\rho_{i}(4, \mathrm{pp} .27-28)$. We shall denote these by $\cap \rho_{i}$ and $\vee \rho_{i}$ respectively, for typographical simplicity. The identity congruence on $S$ will be denoted by ıs.

Let $\mathscr{K}$ denote any one of the symbols $\mathscr{L}, \mathscr{R}, \mathscr{H}, \mathscr{D}, \mathscr{J}$ and let $S$ be an arbitrary semigroup. Corresponding to each $\rho \in \mathscr{C}(S)$ we define an equivalence $\varepsilon(\mathscr{K}, \rho)$ on $S$, in terms of $\mathscr{K}^{S / \rho}$ (Green's equivalence $\mathscr{K}$ on $S / \rho$ ), by prescribing that

$$
(a, b) \in \varepsilon(\mathscr{K}, \rho) \Leftrightarrow(a \rho, b \rho) \in \mathscr{K}^{S / \rho}
$$


and we denote the greatest congruence on $S$ contained in $\varepsilon(\mathscr{K}, \rho)$ by $\mu(\mathscr{K}, \rho)(4$, p. 27$)$. Evidently

$$
(\forall \rho \in \mathscr{C}(S)) \quad \rho \subseteq \mu(\mathscr{K}, \rho) \subseteq \varepsilon(\mathscr{K}, \rho)
$$

Also, it is easily verified that

and hence that

$$
(\forall \rho, \sigma \in \mathscr{C}(S)) \quad \rho \subseteq \sigma \Rightarrow \varepsilon(\mathscr{K}, \rho) \subseteq \varepsilon(\mathscr{K}, \sigma)
$$

$$
(\forall \rho, \sigma \in \mathscr{C}(S)) \quad \rho \subseteq \sigma \quad \Rightarrow \quad \mu(\mathscr{K}, \rho) \subseteq \mu(\mathscr{K}, \sigma) .
$$

These properties will be used without further comment below.

We now define an equivalence $\theta_{\mathscr{K}}$ on $\mathscr{C}(S)$ by the rule that

$$
(\rho, \sigma) \in \theta_{\mathscr{K}} \quad \Leftrightarrow \quad \varepsilon(\mathscr{K}, \rho)=\varepsilon(\mathscr{K}, \sigma) .
$$

This is the central concept in the present investigation. We note immediately that since $\varepsilon(\mathscr{K}, S \times S)=S \times S$, the $\theta_{\mathscr{K}}$-class $(S \times S) \theta_{\mathscr{K}}$ comprises all congruences on $S$ such that $S / \rho$ is $\mathscr{K}$-simple (that is, consists of a single $\mathscr{K}$-class). In particular, $(S \times S) \theta_{\mathscr{H}}$ is the set of all group congruences on $S$.

In preparation for Theorem 1 , which deals with the four cases $\mathscr{K}=\mathscr{L}, \mathscr{R}, \mathscr{H}$ and $\mathscr{J}$, we have

Lemma 1. Let $S$ be a semigroup and let $\mathscr{K}$ denote $\mathscr{L}, \mathscr{R}, \mathscr{H}$ or $\mathscr{Z}$. Then

(i) $(\forall \rho, \sigma \in \mathscr{C}(S)) \quad \rho \subseteq \sigma \subseteq \varepsilon(\mathscr{K}, \rho) \Rightarrow(\rho, \sigma) \in \theta_{\mathscr{H}}$;

(ii) $(\forall \rho \in \mathscr{C}(S)) \mu(\mathscr{K}, \rho)$ is the greatest element in $\rho \theta_{\mathscr{R}}$.

Proof. We establish the result for the case $\mathscr{K}=\mathscr{L}$. The argument is easily adapted to cover each of the remaining cases.

(i) Let $\rho, \sigma \in \mathscr{C}(S)$ and suppose that $\rho \subseteq \sigma \subseteq \varepsilon(\mathscr{L}, \rho)$. Let $(a, b) \in \varepsilon(\mathscr{L}, \sigma)$. Then $(a \sigma, b \sigma) \in \mathscr{L}^{S / \rho}$ and so there exist $x, y \in S^{1}$ such that $(x a, b) \in \sigma$ and $(a, y b) \in \sigma$. Thus $(x a, b) \in \varepsilon(\mathscr{L}, \rho)$ and $(a, y b) \in \varepsilon(\mathscr{L}, \rho)$. This means, in particular, that there exist $u, v \in$ $S^{1}$ such that $(u x a, b) \in \rho$ and $(a, v y b) \in \rho$. Hence $(a \rho, b \rho) \in \mathscr{L}^{S / \rho}$ and so $(a, b) \in \varepsilon(\mathscr{L}, \rho)$. We have thus shown that $\varepsilon(\mathscr{L}, \sigma) \subseteq \varepsilon(\mathscr{L}, \rho)$. But the reverse inclusion holds, since $\rho \subseteq \sigma$. Therefore $\varepsilon(\mathscr{L}, \rho)=\varepsilon(\mathscr{L}, \sigma)$; that is, $(\rho, \sigma) \in \theta_{\mathscr{L}}$.

(ii) Let $\rho \in \mathscr{C}(S)$. Then $\rho \subseteq \mu(\mathscr{L}, \rho) \subseteq \varepsilon(\mathscr{L}, \rho)$ and so, by (i), $(\rho, \mu(\mathscr{L}, \rho)) \in \theta_{\mathscr{L} \text {. Since }}$ $\mu(\mathscr{L}, \rho)$ contains every congruence contained in $\varepsilon(\mathscr{L}, \rho)$, the result follows.

The proof of (i) above closely resembles that of Theorem 13 of (3). In addition, the example cited by Hall $(3$, p. 174) to show that this theorem does not hold for $\mathscr{K}=\mathscr{D}$ also shows that (i) does not hold for $\mathscr{K}=\mathscr{D}$, as we shall demonstrate. Let $S$ be a simple idempotent-free semigroup containing at least two minimal right ideals. (The existence of such semigroups is established in $(\mathbf{1}, \S 8.2)$.) Then it can be shown that $\mathscr{D}(=\mathscr{R})$ is a congruence on $S$ and that $S / \mathscr{D}$ is a nontrivial left zero semigroup (1, Exercise 1 for \$8.2). Hence we have that $\iota_{S} \subseteq \mathscr{D}=\varepsilon\left(\mathscr{D}, \iota_{S}\right)$, while $\left(\iota_{S}, \mathscr{D}\right) \notin \theta_{\mathscr{D}}($ for $\varepsilon(\mathscr{D}, \mathscr{D})=S \times S \neq \mathscr{D})$.

We now come to the first main result.

Theorem 1. Let $S$ be a semigroup and let $\mathscr{K}$ denote $\mathscr{L}, \mathscr{R}, \mathscr{H}$ or $\mathscr{F}$. Then

(i) $\theta_{\mathscr{K}}$ is a complete join congruence on $\mathscr{C}(S)$;

(ii) each $\theta_{\mathscr{K}}$-class is a convex complete join subsemilattice of $\mathscr{C}(S)$. 
Proof. For brevity, we shall denote $\varepsilon(\mathscr{K}, \rho)$ and $\mu(\mathscr{K}, \rho)$ by $\varepsilon(\rho)$ and $\mu(\rho)$ respectively.

(i) We first show that, for any nonempty family $\left(\tau_{i}\right)_{i \in I}$ of congruences on $S$,

$$
\left(\vee \tau_{i}, \vee \mu\left(\tau_{i}\right)\right) \in \theta_{\mathscr{K}}
$$

Let $\left(\tau_{i}\right)_{i \in I}$ be such a family and let $\tau$ denote $\vee \tau_{i}$. Then $\mu\left(\tau_{i}\right) \subseteq \mu(\tau)$ for all $i \in I$ and so $\vee \mu\left(\tau_{i}\right) \subseteq \mu(\tau)$. Also, $\tau \subseteq \vee \mu\left(\tau_{i}\right)$; hence $\varepsilon(\tau) \subseteq \varepsilon\left(\vee \mu\left(\tau_{i}\right)\right)$ and therefore $\mu(\tau) \subseteq \varepsilon\left(\vee \mu\left(\tau_{i}\right)\right)$. Combining these facts, we have that

$$
\vee \mu\left(\tau_{i}\right) \subseteq \mu(\tau) \subseteq \varepsilon\left(\vee \mu\left(\tau_{i}\right)\right)
$$

Thus, by Lemma 1 (i), ( $\left.\vee \mu\left(\tau_{i}\right), \mu(\tau)\right) \in \theta_{\mathscr{K}}$. But, by Lemma 1 (ii), $(\tau, \mu(\tau)) \in \theta_{\mathscr{K}}$. Hence $(\alpha)$ holds.

Now let $\left(\rho_{i}, \sigma_{i}\right)_{i \in I}$ be a nonempty family of elements of $\theta_{\mathscr{K}}$ and let $\rho=\vee \rho_{i}, \sigma=\vee \sigma_{i}$. Since $\varepsilon\left(\rho_{i}\right)=\varepsilon\left(\sigma_{i}\right)$ for all $i \in I$ it follows that $\mu\left(\rho_{i}\right)=\mu\left(\sigma_{i}\right)$ for all $i \in I$ and so $\vee \mu\left(\rho_{i}\right)=$ $\vee \mu\left(\sigma_{i}\right)$. But, from $(\alpha),\left(\rho, \vee \mu\left(\rho_{i}\right)\right) \in \theta_{\mathscr{K}}$ and $\left(\sigma, \vee \mu\left(\sigma_{i}\right)\right) \in \theta_{\mathscr{K}}$. Hence $(\rho, \sigma) \in \theta_{\mathscr{K}}$. Thus $\theta_{\mathscr{K}}$ is a complete join congruence on $\mathscr{C}(S)$.

(ii) It follows from (i) that each $\theta_{\mathscr{H}}$-class is a complete join subsemilattice of $\mathscr{C}(S)$. To complete the proof we show that each $\theta_{\mathscr{Y}}$-class is also convex. Let $\rho, \xi, \sigma \in \mathscr{C}(S)$ be such that $\rho \subseteq \xi \subseteq \sigma$ and $(\rho, \sigma) \in \theta_{\mathscr{K}}$. Then $(\rho \vee \xi, \sigma \vee \xi) \in \theta_{\mathscr{K}}$; that is, $(\xi, \sigma) \in \theta_{\mathscr{K}}$, as required.

Let $S$ and $\mathscr{K}$ be as in the statement of the theorem. Evidently $\varepsilon\left(\mathscr{K}, \iota_{S}\right)=\mathscr{K}\left(=\mathscr{K}^{S}\right)$; hence if $\rho \in \iota_{S} \theta_{\mathscr{K}}$ then $\rho \subseteq \mathscr{K}$. Conversely, if $\rho \in \mathscr{C}(S)$ is such that $\rho \subseteq \mathscr{K}$ then $\iota_{S} \subseteq \rho \subseteq$ $\varepsilon\left(\mathscr{K}, \iota_{S}\right)$ and so, by Lemma 1 (i), $\rho \in \iota_{S} \theta_{\mathscr{K}}$. Thus $\iota_{S} \theta_{\mathscr{K}}$ consists of all congruences contained in $\mathscr{K}$. It follows that $\iota_{S} \theta_{\mathscr{K}}$ is a complete sublattice of $\mathscr{C}(S)$, with least element $\iota_{S}$ and greatest element $\mu\left(\mathscr{K}, \iota_{s}\right)$.

In general, however, a $\theta_{\mathscr{H}}$-class need not be a complete sublattice of $\mathscr{C}(S)$. (To see this we need only consider $(S \times S) \theta_{\mathscr{C}}$, where $S$ is any semigroup that does not possess a least group congruence.) It may even happen that the meet of two elements of a $\theta_{\mathscr{K}}$-class does not itself lie in that $\theta_{\mathscr{K}}$-class - a possibility that is illustrated for $\mathscr{K}=\mathscr{J}$ by the example that follows Theorem 2 below.

We next supplement the results of Lemma 1 and Theorem 1 for the case $\mathscr{K}=\mathscr{H}$ with an extension of a theorem due to Lallement (5).

Lemma 2. For all congruences $\rho$ on a semigroup $S$

$$
\{\xi \in \mathscr{C}(S): \rho \subseteq \xi \subseteq \varepsilon(\mathscr{H}, \rho)\}
$$

is a convex modular complete sublattice of $\mathscr{C}(S)$ contained in $\rho \theta_{\mathscr{H}}$.

Proof. Let $\rho \in \mathscr{C}(S)$ and let $\mathscr{S}(\rho)$ denote the set of all $\xi \in \mathscr{C}(S)$ such that $\rho \subseteq \xi \subseteq$ $\varepsilon(\mathscr{H}, \rho)$. Evidently $\mathscr{S}(\rho)$ is a convex complete sublattice of $\mathscr{C}(S)$, with least element $\rho$ and greatest element $\mu(\mathscr{H}, \rho)$ : also, by Lemma 1 (i), $\mathscr{S}(\rho) \subseteq \rho \theta_{\mathscr{H}}$.

It remains to prove that $\mathscr{P}(\rho)$ is modular. We see easily that $\mathscr{S}(\rho)$ is lattice-isomorphic to the complete sublattice $\mathscr{T}(\rho)$ of $\mathscr{C}(S / \rho)$ consisting of all congruences contained in $\mathscr{H} S / \rho$. But $\mathscr{T}(\rho)$ is modular (5, Théorème 3.2$)$; hence so also is $\mathscr{S}(\rho)$.

In particular, Lemma 2 shows that if a $\theta_{\mathscr{H}}$-class $P$ has a least element then $P$ is itself a convex complete modular sublattice of $\mathscr{C}(S)$.

For the rest of the discussion we restrict ourselves to the case of regular semigroups. 
The following argument, based on a result in (3), shows that the conclusions of Lemma 1 and Theorem 1 hold when $S$ is a regular semigroup and $\mathscr{K}=\mathscr{D}$. It suffices to establish assertion (i) of Lemma 1 for such $S$ and $\mathscr{K}$. Accordingly, suppose that $S$ is a regular semigroup and that $\rho, \sigma \in \mathscr{C}(S)$ are such that $\rho \subseteq \sigma \subseteq \varepsilon(\mathscr{D}, \rho)$. Let $(a, b) \in \varepsilon(\mathscr{D}, \sigma)$. Then $(a \sigma, b \sigma) \in \mathscr{D}^{S / \sigma}$. Hence, by $(3$, Theorem 10$)$, there exist $c, d \in S$ such that $(c, d) \in \mathscr{D}^{S}$, $(a, c) \in \sigma$ and $(b, d) \in \sigma$. Consequently, $(c \rho, d \rho) \in \mathscr{D}^{S / \rho},(a \rho, c \rho) \in \mathscr{D}^{S / \rho}$ and $(b \rho, d \rho) \in$ $\mathscr{D}^{S / \rho}$, from which it follows that $(a \rho, b \rho) \in \mathscr{D}^{S / \rho}$. Thus $(a, b) \in \varepsilon(\mathscr{D}, \rho)$. This shows that $\varepsilon(\mathscr{D}, \sigma) \subseteq \varepsilon(\mathscr{D}, \rho)$. But the reverse inclusion holds since $\rho \subseteq \sigma$ and so $(\rho, \sigma) \in \theta_{\mathscr{D}}$.

A substantially stronger result than Theorem 1 holds for regular semigroups in the three cases $\mathscr{K}=\mathscr{L}, \mathscr{R}, \mathscr{H}$.

Theorem 2. Let $S$ be a regular semigroup and let $\mathscr{K}$ denote $\mathscr{L}, \mathscr{R}$ or $\mathscr{H}$. Then

(i) $\theta_{\mathscr{K}}$ is a complete lattice congruence on $\mathscr{C}(S)$;

(ii) each $\theta_{\mathscr{K}}$-class is a convex complete sublattice of $\mathscr{C}(S)$ and is modular if $\mathscr{K}=\mathscr{H}$.

Proof. Again, for brevity, we denote $\varepsilon(\mathscr{K}, \rho)$ and $\mu(\mathscr{K}, \rho)$ by $\varepsilon(\rho)$ and $\mu(\rho)$ respectively.

We begin by showing that, for an arbitrary nonempty family $\left(\tau_{i}\right)_{i \in I}$ of congruences on $S$,

$$
\mu\left(\cap \tau_{i}\right)=\cap \mu\left(\tau_{i}\right)
$$

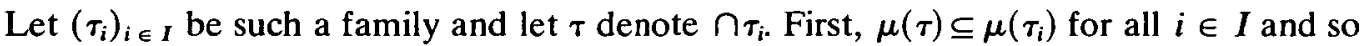
$\mu(\tau) \subseteq \cap \mu\left(\tau_{i}\right)$. We now establish the reverse inclusion.

Consider the case $\mathscr{K}=\mathscr{L}$. Let $(a, b) \in \cap \mu\left(\tau_{i}\right)$ and let $a^{\prime}, b^{\prime}$ denote inverses in $S$ of $a, b$ respectively. Now $(a, b) \in \cap \varepsilon\left(\tau_{i}\right)$ and so $\left(a \tau_{i}, b \tau_{i}\right) \in \mathscr{L}^{S / \tau_{i}}$ for all $i \in I$. Hence $\left(a b^{\prime} b, a\right) \in$ $\tau_{i}$ and $\left(b a^{\prime} a, b\right) \in \tau_{i}$ for all $i \in I$. It follows that $\left(a b^{\prime} b, a\right) \in \tau$ and $\left(b a^{\prime} a, b\right) \in \tau$. Consequently, $(a \tau, b \tau) \in \mathscr{L}^{S / \tau}$; that is, $(a, b) \in \varepsilon(\tau)$. We have thus shown that $\cap \mu\left(\tau_{i}\right) \subseteq \varepsilon(\tau)$. Hence $\cap \mu\left(\tau_{i}\right) \subseteq \mu(\tau)$. A similar argument shows that this result also holds for the cases $\mathscr{K}=\mathscr{R}$ and $\mathscr{K}=\mathscr{H}$. Thus $(\beta)$ holds.

From Theorem $1, \theta_{\mathscr{K}}$ is a complete join congruence on $\mathscr{C}(S)$ and each $\theta_{\mathscr{K}}$-class is a convex complete join subsemilattice of $\mathscr{C}(S)$. Now let $\left(\rho_{i}, \sigma_{i}\right)_{i \in I}$ be a nonempty family of elements of $\theta_{\mathscr{K}}$. For all $i \in I, \varepsilon\left(\rho_{i}\right)=\varepsilon\left(\sigma_{i}\right)$ and so $\mu\left(\rho_{i}\right)=\mu\left(\sigma_{i}\right)$. Hence $\cap \mu\left(\rho_{i}\right)=\cap \mu\left(\sigma_{i}\right)$. Thus, from $(\beta), \mu(\rho)=\mu(\sigma)$, where $\rho=\cap \rho_{i}$ and $\sigma=\cap \sigma_{i}$. But $(\rho, \mu(\rho)) \in \theta_{\mathscr{F}}$ and $(\sigma, \mu(\sigma)) \in \theta_{\mathscr{K}}$, by Lemma 1(ii). Hence $(\rho, \sigma) \in \theta_{\mathscr{F}}$. This shows that $\theta_{\mathscr{K}}$ is also a complete meet congruence on $\mathscr{C}(S)$. It follows that $\theta_{\mathscr{K}}$ is a complete lattice congruence on $\mathscr{C}(S)$ and that each $\theta_{\mathscr{K}}$-class is a convex complete sublattice of $\mathscr{C}(S)$. Moreover, from Lemma 2, if $\mathscr{K}=\mathscr{H}$ then each $\theta_{\mathscr{K}}$-class is modular. This completes the proof.

The following example, again taken from (3), shows that we cannot extend the hypotheses of Theorem 2 to include the cases $\mathscr{K}=\mathscr{D}$ or $\mathscr{K}=\mathscr{g}$. Let $B$ denote the bicyclic semigroup: specifically, take $B=N \times N$, where $N$ is the set of all non-negative integers and multiplication is given by

$$
(m, n)(p, q)=(m-n+h q-p+t), \quad t=\max (n, p) .
$$

Now let $S$ denote the subset

$$
\{((m, n),(p, q)): m+p=n+q\}
$$


of the direct product $B \times B$ (an inverse semigroup). Then $S$ is a (full) inverse subsemigroup of $B \times B, \mathscr{D}=\mathscr{J}$ on $S$ and $S$ has infinitely many $\mathscr{D}$-classes $(3, \S 6)$. (In fact, $S \cong \mathscr{F} \mathscr{F}_{1}^{1}$, where $\mathscr{F} \Phi_{1}$ is the free inverse semigroup of rank 1.) Next, let $\psi_{1}$ and $\psi_{2}$ denote the homomorphisms of $S$ onto $B$ defined by the rules

$$
((m, n),(p, q)) \psi_{1}=(m, n), \quad((m, n),(p, q)) \psi_{2}=(p, q)
$$

and let $\rho_{i}=\psi_{i} \circ \psi_{i}^{-1}(i=1,2)$. Clearly $S / \rho_{1} \cong S / \rho_{2} \cong B$ and $\rho_{1} \cap \rho_{2}=\iota_{s}$. Hence, since $B$ is bisimple [simple] while $S$ is not, it follows that $(S \times S) \theta_{\mathscr{D}}\left[=(S \times S) \theta_{\mathscr{J}}\right]$ is not closed under intersection.

To conclude, we restrict our attention to the case $\mathscr{K}=\mathscr{H}$. Let $S$ be a regular semigroup and let $E$ denote its set of idempotents. As in (6), we define a relation $\theta$ on $\mathscr{C}(S)$ as follows:

$$
(\rho, \sigma) \in \theta \Leftrightarrow \rho \cap(E \times E)=\sigma \cap(E \times E) .
$$

Then we have

Lemma 3. Let $S$ be a regular semigroup. Then $\theta_{\mathscr{H}}=\theta$ on $\mathscr{C}(S)$.

Proof. Let $E$ denote the set of all idempotents of $S$. For all $\tau \in \mathscr{C}(S)$, denote $\tau \cap(E \times E)$ by $\left.\tau\right|_{E}$ and $\varepsilon(\mathscr{H}, \tau)$ by $\varepsilon(\tau)$.

First, suppose that $(\rho, \sigma) \in \theta_{\mathscr{F}}$ Let $\left.(e, f) \in \rho\right|_{\mathrm{E}}$. Then $(e, f) \in \varepsilon(\rho)$ and so $(e, f) \in \varepsilon(\sigma)$. Thus $(e \sigma, f \sigma) \in \mathscr{X}^{S / \sigma}$. Hence, since $e \sigma$ and $f \sigma$ are idempotents in $S / \sigma, e \sigma=f \sigma$ and so $\left.(e, f) \in \sigma\right|_{E}$. Thus $\left.\left.\rho\right|_{E} \subseteq \sigma\right|_{E \cdot}$. Similarly, $\left.\left.\sigma\right|_{E} \subseteq \rho\right|_{E}$. Hence $\left.\rho\right|_{E}=\left.\sigma\right|_{E}$; that is, $(\rho, \sigma) \in \theta$.

Conversely, suppose that $(\rho, \sigma) \in \theta$. Let $(a, b) \in \varepsilon(\rho)$ and let $a^{\prime}, b^{\prime}$ denote inverses in $S$ of $a, b$ respectively. Since $(a \rho, b \rho) \in \mathscr{H}^{S / \rho}$ it follows, in particular, that $\left(a b^{\prime} b, a\right) \in \rho$. Hence,

$$
\text { (1) }\left(a^{\prime} a b^{\prime} b, a^{\prime} a\right) \in \rho \text {. }
$$

Now, by Lallement's lemma (5; 4, Lemma II 4.6) there exist $e \in E$ and $x \in S$ such that

$$
\text { (2) }\left(a^{\prime} a b^{\prime} b, e\right) \in \rho, \text { (3) } e=x a^{\prime} a b^{\prime} b \text {. }
$$

By (1) and (2), $\left.\left(e, a^{\prime} a\right) \in \rho\right|_{E}$ and so, by hypothesis, $\left.\left(e, a^{\prime} a\right) \in \sigma\right|_{E}$. Hence $(a e, a) \in \sigma$ and therefore, by (3), $\left(a x a^{\prime} a b^{\prime} b, a\right) \in \sigma$. Thus $L_{a \sigma} \leqq L_{b \sigma}$ in $S / \sigma$. Interchanging $a$ and $b$, we see that $L_{b \sigma} \leqq L_{a \sigma}$. It follows that $(a \sigma, b \sigma) \in \mathscr{L}^{S / \sigma}$. A dual argument shows that $(a \sigma, b \sigma) \in$ $\mathscr{R}^{S / \sigma}$. Hence $(a \sigma, b \sigma) \in \mathscr{H}^{S / \sigma}$; that is, $(a, b) \in \varepsilon(\sigma)$. We have thus proved that $\varepsilon(\rho) \subseteq$ $\varepsilon(\sigma)$. Similarly, $\varepsilon(\sigma) \subseteq \varepsilon(\rho)$. Hence $\varepsilon(\rho)=\varepsilon(\sigma)$ and so $(\rho, \sigma) \in \theta_{\mathscr{x}}$.

Combining Lemma 3 with Theorem 2 for the case $\mathscr{K}=\mathscr{H}$, we obtain the following well-known theorem on $\theta$-classes established by Reilly and Scheiblich (6), Scheiblich (7) and Hall (2).

Theorem 3. Let $S$ be a regular semigroup. Then

(i) $\theta$ is a complete lattice congruence on $\mathscr{C}(S)$;

(ii) each $\theta$-class is a convex modular complete sublattice of $\mathscr{C}(S)$.

Note added in proof. Suppose that $S$ and $\mathscr{K}$ are as in Theorem 1 . Then, for all $\rho \in \mathscr{C}(S), \mu(\mathscr{K}, \rho)$ is the greatest element in $\rho \theta_{\mathscr{K}}$. Thus the mapping $\rho \mapsto \mu(\mathscr{K}, \rho)$ is a closure operation on $\mathscr{C}(S)$ and so the set of greatest elements of the $\theta_{\mathscr{K}}$-classes is a complete meet 
subsemilattice of $\mathscr{C}(S)$. The same conclusions hold if $S$ is a regular semigroup and $\mathscr{K}=\mathscr{D}$.

For $S$ and $\mathscr{K}$ as in Theorem $2, \theta_{\mathscr{K}}$ is a complete lattice congruence on $\mathscr{C}(S)$ and it follows that the set of least elements of the $\theta_{\mathscr{K}}$-classes is a complete join subsemilattice of $\mathscr{C}(S)$. I am grateful to Dr. T. E. Hall for pointing out the corresponding result for $\theta$-classes.

\section{REFERENCES}

(1) A. H. Clifford and G. B. PRESTON, The algebraic theory of semigroups, Math. Surveys of the Amer. Math. Soc. 7 (Providence, R.I., 1961 (Vol. I) and 1967 (Vol. II)).

(2) T. E. HALL, On the lattice of congruences on a regular semigroup, Bull. Australian Math. Soc. 1 (1969), 231-235.

(3) T. E. HALL, Congruences and Green's relations on regular semigroups, Glasgow Math. J.13 (1972), 167-175.

(4) J. M. HowIE, An introduction to semigroup theory, London Math. Soc. monographs 7 (London, 1976).

(5) G. LALLEMENT, Congruences et équivalences de Green sur un demi-groupe régulier, C.R. Acad. Sc. Paris (Sér. A) 262 (1966), 613-616.

(6) N. R. ReIlly and H. E. SCHEIBLICH, Congruences on regular semigroups, Pacific J. Math. 23 (1967), 349-360.

(7) H. E. SCHEIBLICH, Certain congruence and quotient lattices related to completely 0 -simple and primitive regular semigroups, Glasgow Math. J. 10 (1969), 21-24.

DEPARTMENT OF MATHEMATICS

UNIVERSITY OF GLASGOW

GLASGOW, G12 8QW

SCOTLAND 